\title{
QUANTIFICAÇÃO DE BIOMASSA E ESTIMATIVA DE ESTOQUE DE CARBONO DE INDIVÍDUOS DE AROEIRA EM UM FRAGMENTO DE FLORESTA ESTACIONAL DECIDUAL
}

Renan Augusto Miranda MATIAS ${ }^{1}$

Bruno Silva FERREIRA ${ }^{2}$

Thelma Shirlen SOARES ${ }^{2}$

${ }^{1}$ UFG - Regional Jataí. renanmatias@ hotmail.com

${ }^{2}$ UFG - Regional Jataí. brunoferreira_florestal@hotmail.com

${ }^{3}$ UFG - Regional Jataí. thelsoares@ gmail.com

Recebido em: 18/10/2016 - Aprovado em: 20/11/2017 - Disponibilizado em: 30/12/2017

\begin{abstract}
RESUMO
Este estudo teve como objetivo caracterizar a estrutura paramétrica e o estoque de carbono de indivíduos dearoeira (Myracrodruon urundeuvaAll.) presente em um fragmento de floresta estacional decidual localizadoem Jataí, GO. Para avaliação do fragmento, foi realizado um censo, mensurando todos os indivíduos com cap (circunferência a 1,3 m do solo) $\geq 15,7 \mathrm{~cm}$ e georreferenciando-os. Após estabelecer as classes diamétricas,mensurou-se a altura total de 10 árvores por classe para o ajuste e seleção de um modelo hipsométrico e obtenção do volume, biomassae estoque de carbono. Foram identificados 1807 indivíduos distribuídos em toda a área do fragmento,os quais estão distribuídos em classes diamétricas com distribuição sigmoidal, com exceção para as árvores da primeira classe. O diâmetro médio das árvores foi de $15,5 \mathrm{~cm}$. A equação hipsométrica selecionada para o povoamento foi $\ln (H)=2,85102-4,66042 \mathrm{dap}^{-1}$, com $\mathrm{R}^{2}=77,8 \%$ e erro padrão residual de $\pm 1,6 \mathrm{~m}$. O volume de madeira oriundo dos indivíduos de aroeira mensurados foide $45 \mathrm{~m}$ /ha, com produção de 40 t/ha de biomassa e estoque de 20 t/ha de carbono.
\end{abstract}

Palavra-chave: Myracrodruon urundeuva All.; fragmento florestal; censo florestal; volume; alometria.

\begin{abstract}
This study aimed to characterize the parametric structure and carbon stock of individuals of aroeira (Myracrodruon urundeuva All.) present in a fragment Decidual Seasonal Forest located in Jataí, Goiás, Brazil. For evaluation of the fragment, a Forest Census was conducted, measuring up all individuals with $c b h$ (circumference at breast height at 1.30 $\mathrm{m}) \geq 15.7 \mathrm{~cm}$. All trees measured were georeferenced. After establishing the diameter classes, measured to total height of 10 trees per class for setting and selecting a hypsometric model and obtain the volume, biomass and carbon stock. 1807 individuals were identified distributed in the area, which are divided into diameter classes with sigmoidal distribution, except for the trees of the first class. The average diameter tree was $15.5 \mathrm{~cm}$. The hypsometric equation selected for the settlement was $\ln (\mathrm{H})=2.85102-4.66042 \mathrm{dap}^{-1}$ with $\mathrm{R}^{2}=77.78 \%$ and residual standard error of $\pm 1.6 \mathrm{~m}$. The volume of wood obtained was $45 \mathrm{~m}^{3} /$ ha with production of $40 \mathrm{t} / \mathrm{ha}$ of biomass and of $20 \mathrm{t} / \mathrm{ha}$ of stock carbon.
\end{abstract}

Keywords:Myracrodruon urundeuva All.; forest fragment; forest census; volume; allometry.

\section{INTRODUÇÃO}

\section{A aroeira (Myracrodruon urundeuva}

All.), também conhecida por aroeira-do-sertão, aroeira-do-campo, aroeira-preta, aroeiraverdadeira, dentre outros, pertence à família Anacardiaceae e é uma árvore decídua, heliófila, seletiva xerófila, característica de terrenos secos e rochosos (LORENZI, 2002). É uma espécie nativa do Brasil, com ampla distribuição geográfica ocorrendo nas regiões Nordeste, Sudeste, Centro-oeste e do Sul (SANTIN e LEITÃO FILHO, 1991).

Uma das características sociológicas da aroeira é que a mesma apresenta regeneração por rebrota em vegetação secundária, com grande quantidade de plantas de todas idades, formando algumas vezes bosques quase puros (GUARIM NETO, 1986). 
É uma espécie de grande importância econômica em função do aproveitamento da madeira (construção civil, postes, mourões, móveis, dormentes), da extração de taninos e da utilização na farmacologia (CARVALHO, 2003; CARLINI et al., 2010).

A aroeira tornou-se escassa com todas as áreas de ocorrência devido ao extrativismo (PAULA e ALVES, 1997). Por isso, a espécie está na Lista Oficial das Espécies da Flora Brasileira Ameaçadas de Extinção, na classe vulnerável, desde 1992 (BRASIL, 2008).

Vários pesquisadores relatam que cerca de $25 \%$ das espécies vegetais do planeta poderão entrar em perigo de extinção até meados do ano de 2040 (FERNANDES, 2002). Desta forma, estudos sobre espécies ameaçadas de extinção, como o caso da aroeira, são uteis para subsidiar programas de manutenção das mesmas em áreas naturais, além de fornecer parâmetros para o manejo da espécie.

Neste contexto, o objetivo deste estudo foi caracterizar a estrutura paramétrica e o estoque de carbono da espécie aroeira (Myracrodruon urundeuvaAll.)em um fragmento de Floresta Estacional Decidual localizado em Jataí - GO.

\section{MATERIAL E MÉTODOS}

O fragmento florestal estudado está situado a $17^{\circ} 56^{\prime} \mathrm{S}$ e $51^{\circ} 43^{\prime} \mathrm{W}$, na Regional Jataí da Universidade Federal de Goiás, localizado no município de Jataí, região sudoeste do Estado de Goiás (Figura 1). Possui área de 8,2 ha e altitude média de $629 \mathrm{~m}$. A vegetação da área é classificada como floresta estacional decidual (BRASIL, 2012) e o solo como Latossolo Vermelho Distrófico (EMBRAPA, 1999).

A região apresenta clima do tipo $A w$ (clima tropical com inverno seco) segundo a classificação de Köppen, identificado como clima tropical de savana. A temperatura média anual é de $23,7{ }^{\circ} \mathrm{C}$ e a precipitação anual média de 1644,9 mm (INMET, 2013).

Figura 1 - Localização da área de estudo no município de Jataí, GO.

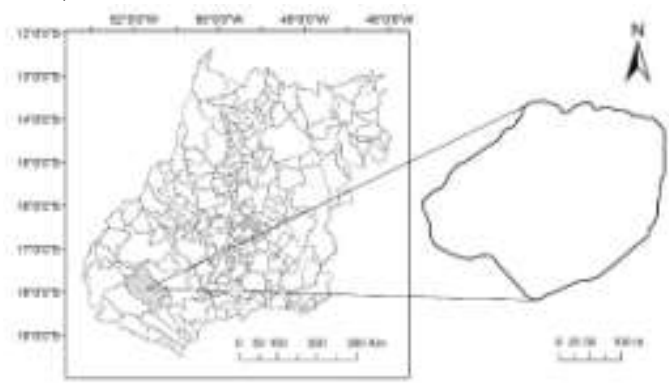

O fragmento é circundado por ambientes antropizados, com áreas de pastagem ao leste $\mathrm{e}$ sul, uma Estação de Tratamento de Esgoto (E.T.E.) na porção norte e um curso d'agua (Rio Claro) a oeste.

O levantamento dos indivíduos de aroeira foi realizado por meio de censo florestal, ou inventário a $100 \%$. Todos os indivíduos com cap (circunferência a 1,3 $\mathrm{m}$ do solo) $\geq 15,7 \mathrm{~cm}$ foram identificados, georreferenciados e tiveram o cap mensurado.

Para conhecer a estrutura diamétrica do fragmento, representada pela distribuição de árvores em classes de diâmetros, as medidas de cap foram convertidas em dap (diâmetro a 
1,3 $\mathrm{m}$ do solo) e agrupadas pelo método estatístico, conforme recomendado por Scolforo e Thiersch (2004), ilustrando a dispersão das árvores do povoamento.

Para cada classe diamétrica estabelecida, foram mensuradas as alturas de 10 árvores para estimativa de uma equação hipsométrica para o povoamento. Os modelos ajustados são apresentados na Tabela 1 .

Tabela 1 - Modelos testados para estimar a altura total de árvores individuais de aroeira.

\begin{tabular}{ll}
\hline Modelo & Relação funcional* \\
\hline Linha reta & $H=\beta_{1}+\beta_{2} d a p+\varepsilon i$ \\
Parabólico & $H=\beta_{0}+\beta_{1} d a p+\beta_{2} d a p^{2}+\varepsilon i$ \\
Stoffel & $\ln (H)=\beta_{0}+\beta_{1} \ln (d a p)+\ln \left(\varepsilon_{i}\right)$ \\
Curtis & $\ln (H)=\beta_{0}+\beta_{1} d a p^{1}+\ln \left(\varepsilon_{i}\right)$ \\
\hline
\end{tabular}

* Em que: $H=$ altura total, dap = diâmetro a $1,3 \mathrm{~m}$ do solo, $\beta_{\text {is }}=$ coeficientes dos modelos.

Os modelos hipsométricos foram ajustados pelo método dos mínimos quadrados conforme descrito por Draper e Smith (1998).A avaliação e comparação dos modelos foram realizadas de acordo com as seguintes estatísticas: coeficiente de determinação $\left(\mathrm{R}^{2}\right)$, erro padrão da estimativa $\left(\mathrm{S}_{\mathrm{yx} \%}\right)$ e distribuição gráfica dos resíduos percentuais. Para fins de comparação dos modelos logarítmicos com os não-logarítmicos foi recalculado o erro padrão da estimativa e o coeficiente de determinação conforme recomendado por Machado et al. (2004).

Com os dados do dap e das estimativas das alturas do povoamento, obteve-se o volume empregando um fator de forma 0,7 , de acordo com SEMA (2007).
A partir dos dados obtidos, foram calculadas as seguintes variáveis dendrométricas para a aroeira nas diferentes classes diamétricas: diâmetro médio aritmético e quadrático, diâmetro mínimo e máximo, altura média aritmética e área basal, conforme Scolforo e Thiersch (2004) e Machado e Figueredo Filho (2009).

Também foi calculado o estoque de carbono armazenado (considerando a altura total) nas árvores de aroeira presentes no fragmento. Foi considerado um valor de densidade básica igual a $0,86 \mathrm{~g} / \mathrm{cm}^{3}$ conforme Siqueira et al. (2013). A seguir, estimou-se a biomassa da madeira de cada árvore pela multiplicação da densidade básica média pelos volumes totais com casca, conforme a expressão:

$$
\mathrm{B}=\overline{\mathrm{d}} \times \mathrm{V}
$$

em que:

$\mathrm{B}=$ biomassa da árvore, em $\mathrm{kg}$;

$\bar{d}$ = densidade básica média, em $\mathrm{kg} / \mathrm{m}^{3}$; $\mathrm{V}=$ volume total com casca estimado de árvores individuais, $\mathrm{em}^{3}$.

A estimativa do carbono estocado na biomassa das árvores foi obtida pela multiplicação das estimativas de biomassa obtidas pelo fator 0,5 , conforme Soares et al. (2006), considerando-se que a biomassa seca contém aproximadamente $50 \%$ de carbono.

\section{RESULTADOS E DISCUSSÃO}

Foram mensurados um total de 1807 indivíduos de aroeira, com diâmetro médio de 
$15,5 \mathrm{~cm}$ e desvio padrão de $\pm 7,0 \mathrm{~cm}$, os quais estão distribuídos em toda a área do fragmento (Figura 2).

Figura 2 - Distribuição dos indivíduos de aroeira com $c a p \geq 15,7 \mathrm{~cm}$ no fragmento estudado, Jataí, GO.

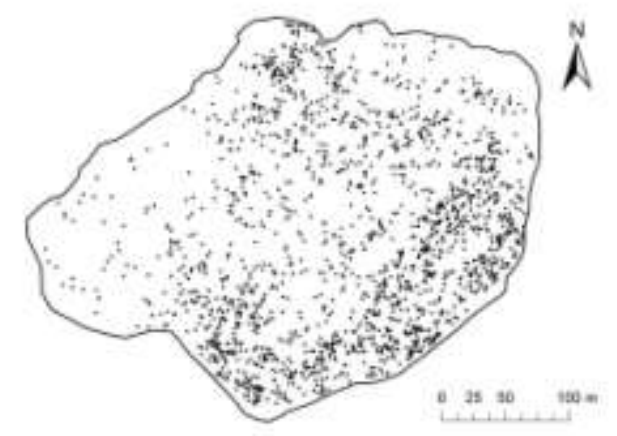

Os indivíduos mensurados foram agrupados em sete classes diamétricas conforme Tabela 2, sendo que a maior quantidade de indivíduos foi encontrada nas classes de menor diâmetro.

Tabela 2 - Distribuição diamétrica da aroeira na área em estudo, Jataí, GO.

\begin{tabular}{cccc}
\hline $\begin{array}{c}\text { Classe } \\
\text { diamétrica }\end{array}$ & $\begin{array}{c}\text { Centro } \\
\text { de classe }\end{array}$ & $\begin{array}{c}\text { Frequência } \\
\left(\mathbf{n}^{\mathbf{0}} \text { ind. }\right)\end{array}$ & $\begin{array}{c}\text { Frequência } \\
\text { /ha } \\
\left(\mathbf{n}^{\mathbf{o}} \text { ind. }\right)\end{array}$ \\
\hline $5,0 \neg 12,0$ & 8,5 & 636 & 78 \\
$12,0 \neg 18,9$ & 15,5 & 717 & 87 \\
$18,9 \neg 25,9$ & 22,4 & 304 & 37 \\
$25,9 \neg 32,9$ & 29,4 & 106 & 13 \\
$32,9 \neg 39,9$ & 36,4 & 33 & 4 \\
$39,9 \neg 46,9$ & 43,4 & 6 & 1 \\
$46,9 \neg 53,8$ & 50,4 & 5 & 1 \\
\hline \multicolumn{5}{c}{ Total } & & 1807 & 221 \\
\hline
\end{tabular}

Neste fragmento, foi encontrado um total de 221 ind./ha. Machado Júnior (2007) estudando um fragmento com monodominância de aroeira no Vale do Rio Doce, MG, registrou a presença de 776 ind./ha.

Observa-se na Figura 3 que os indivíduos de aroeira apresentaram comportamento similar ao "J invertido", ou seja, distribuição sigmoidal, característico de florestas inequiâneas, exceto para a primeira classe, indicando, conforme Scolforo (1998), que há problemas na sua regeneração natural. Tal fato ocorreu, provavelmente, por ser uma área antropizada, e ao longo do estudo, observou-se a presença de gado em sua bordadura, o quepode ter impedido o desenvolvimento do banco de plântulas. Comportamento similar foi observado por Oliveira (2011) em florestas com monodominância de aroeira no município de Tumiritinga, MG.

Figura 3 - Número de indivíduos de aroeira por classe diamétrica, em um fragmento florestal em Jataí, GO.

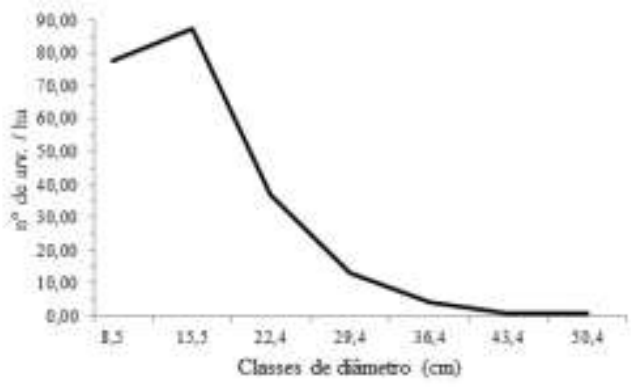

$\mathrm{Na}$ Tabela 3 encontram-se os valores por classe diamétrica das variáveis dendrométricas calculadas para os indivíduos de aroeira presentes no fragmento.

Tabela 3 - Variáveis dendrométricas calculadas para os indivíduos de aroeira com cap $\geq 15,7 \mathrm{~cm}$, em um fragmento florestal em Jataí, GO**

\begin{tabular}{|c|c|c|c|c|c|c|}
\hline $\begin{array}{c}\text { Classe } \\
\text { diamétrica }\end{array}$ & $\begin{array}{l}d_{\min } \\
(\mathbf{c m})\end{array}$ & $\begin{array}{l}d_{\max } \\
(\mathbf{c m})\end{array}$ & $\begin{array}{c}\bar{d} \\
(\mathbf{c m})\end{array}$ & $\begin{array}{c}d g \\
(\mathbf{c m})\end{array}$ & $\begin{array}{l}\bar{H} \\
(\mathbf{m})\end{array}$ & $\begin{array}{c}G \\
\left(\mathbf{m}^{2} / \mathbf{h a}\right)\end{array}$ \\
\hline $5,0 \neg 12,0$ & 5,1 & 11,9 & 9,0 & 9,2 & 10,1 & 0,5159 \\
\hline $12,0 \neg 18,9$ & 12,0 & 18,9 & 15,2 & 15,3 & 12,7 & 1,5959 \\
\hline $18,9 \neg 25,9$ & 19,0 & 25,8 & 21,7 & 21,8 & 13,9 & 1,3922 \\
\hline $25,9 \neg 32,9$ & 25,9 & 32,6 & 28,8 & 28,8 & 14,7 & 0,8427 \\
\hline $32,9 \neg 39,9$ & 33,1 & 39,6 & 35,7 & 35,9 & 15,2 & 0,4074 \\
\hline $39,9 \neg 46,9$ & 39,9 & 45,0 & 42,3 & 42,3 & 15,5 & 0,1027 \\
\hline $46,9 \neg 53,8$ & 47,6 & 50,9 & 50,4 & 49,8 & 15,8 & 0,1189 \\
\hline
\end{tabular}


Em relação ao ajuste dos modelos hipsométricos, o um melhor ajuste foi obtido para o modelo de Curtis, que apresentou maior coeficiente de determinação $(77,8 \%)$, menor erro padrão de estimativa $( \pm 1,64 \mathrm{~m})$,um melhor padrão de distribuição dos resíduos em relação ao dap, sendo o modelo apropriado para a estimativa das alturas dos indivíduos de aroeira no fragmento estudado. As estatísticas dos modelos ajustados são apresentadas naTabela 4 e Figura4.

Tabela 4 - Coeficiente de determinação e erro padrão da estimativa dos modelos hipsométricos ajustados para aroeira em um fragmento florestal em Jataí, GO.

\begin{tabular}{lccccc}
\hline \multirow{2}{*}{ Modelo } & \multicolumn{3}{c}{ Parâmetros } & \multirow{2}{*}{$\mathbf{R}^{2}(\boldsymbol{\%})$} & \multirow{2}{*}{$\mathbf{S}_{\mathbf{x y}}(\mathbf{m})$} \\
\cline { 2 - 5 } & $\boldsymbol{\beta}_{\mathbf{0}}$ & $\boldsymbol{\beta}_{\mathbf{1}}$ & $\boldsymbol{\beta}_{\mathbf{2}}$ & & \\
\hline Linha reta & 10,16394 & 0,14171 & - & 64,9 & $\pm 2,0$ \\
Parabólico & 6,05283 & 0,55086 & $-0,00803$ & 77,1 & $\pm 1,7$ \\
Curtis & 2,85102 & $-4,66042$ & - & 77,8 & $\pm 1,6$ \\
Stoffel & 1,71773 & 0,28548 & - & 72,7 & $\pm 1,8$ \\
\hline
\end{tabular}

Figura 4 - Distribuição gráfica dos resíduos para os modelos hipsométricos ajustados para aroeira em um fragmento florestal em Jataí, GO.

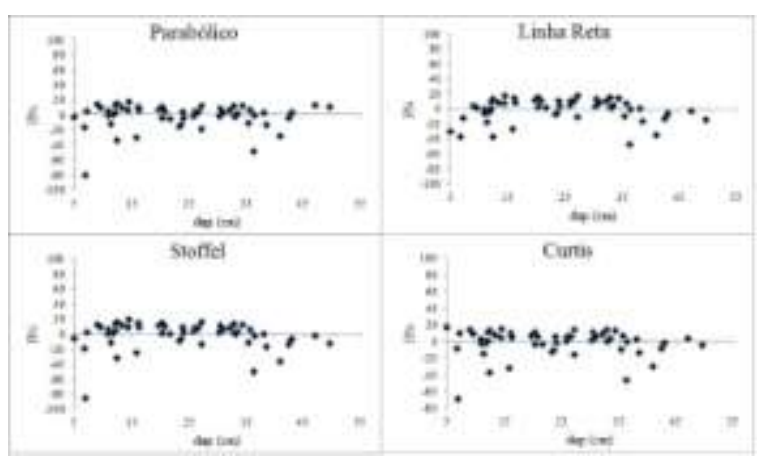

O fragmento apresentou volume total de $381,4968 \mathrm{~m}^{3}$ deindivíduos dearoeira com cap $\geq 15,7 \mathrm{~cm}$,o que equivale a 46,5241 $\mathrm{m}^{3} / \mathrm{ha}$ (Tabela 5).O volume obtido foi $81,1 \%$, superior ao encontrado porRocha (2011) estudando a vegetação lenhosa com dap $\geq 5,0$ $\mathrm{cm}$ em um fragmento de floresta estacional decidual, no município de Campos Belos, GO.
Tabela 5 - Volume e biomassa dos indivíduos de aroeira, por classe diamétrica, em um fragmento florestal em Jataí, GO.

\begin{tabular}{|c|c|c|c|}
\hline Classes diamétricas & $\begin{array}{l}\begin{array}{l}\text { Volume } \\
\left(\mathbf{m}^{3} / \mathbf{h a}\right)\end{array} \\
\end{array}$ & $\begin{array}{c}\text { Biomassa } \\
\text { (t/ha) }\end{array}$ & $\begin{array}{c}\text { Carbono } \\
\text { (t/ha) }\end{array}$ \\
\hline $5,0 \neg 12,0$ & 3,8314 & 3,2950 & 1,6475 \\
\hline $12,0 \neg 18,9$ & 14,3462 & 12,3377 & 6,1689 \\
\hline $18,9 \neg 25,9$ & 13,4642 & 11,5792 & 5,7896 \\
\hline $25,9 \neg 32,9$ & 8,6868 & 7,4707 & 3,7353 \\
\hline $32,9 \neg 39,9$ & 4,1717 & 3,5876 & 1,7938 \\
\hline $39,9 \neg 46,9$ & 0,9502 & 0,8172 & 0,4086 \\
\hline $46,9 \neg 53,8$ & 1,0736 & 0,9233 & 0,4617 \\
\hline Total & 46,5241 & 40,0100 & 20,0053 \\
\hline
\end{tabular}

Verificou-se que a aroeiraencontra-se presente de modo expressivo na área, uma vez que apresentou volume superior a outras tipologias amostradas para um mix de espécies. Scolforo et al. (2008) encontraramdiferentes valores inventariando florestas estacionais deciduais no Estado de Minas Gerais, obtendo volumes variando de $56,46 \mathrm{~m}^{3} / \mathrm{ha}$ a $338,80 \mathrm{~m}^{3} / \mathrm{ha}$, considerando todas as árvores com dap $\geq 5,0 \mathrm{~cm}$ presentes nas áreas inventariadas.

Em relação à distribuição do volume nas diferentes classes diamétricas, verificou-se que cerca de 78,45\% dovolume está presentenas classes entre $12,0 \mathrm{~cm}$ e $32,9 \mathrm{~cm}$, contando com grande quantidade de indivíduos, mas com menor dimensão emdiâmetro. As classes de diâmetro entre $39,9 \mathrm{~cm}$ e $53,8 \mathrm{~cm}$ possuem pouca representatividade no volume total, $4,35 \%$, uma vez que apresentam menor número de indivíduos.

Os resultados médios dos estoques de biomassa e de carbono para os indivíduos de aroeira com $c a p \geq 15,7 \mathrm{~cm}$ foram de 40,0t/ha e 20,0 t/ha, respectivamente (Tabela 5). Se levado em conta toda extensão do fragmento, 
totaliza-se $328,08 \mathrm{t}$ de biomassa e cerca de 164,04 t de carbono estocado.

Os valores obtidos para o estoque de biomassa e de carbono foram superiores aos relatados por Rocha (2011) e Bruzinga et al. (2012) e para a mesma espécie.Rocha (2011), avaliando o estoque de biomassa verde e de carbono em uma floresta estacional decidual em Campos Belos, GO, obteve valores de 8,249 t/ha e 1,951 t/ha, respectivamente. JáBruzinga et al. (2012), quantificando o estoque de biomassa carbono em uma área de floresta estacional semidecidual em regeneração na microrregião de AlmenaraMG, obtiveram valores de 0,1857 t/ha de biomassa e 0,0920 t/ha de carbono.

\section{CONCLUSÕES}

O levantamento dos indivíduos de aroeira com cap $\geq 15,7 \mathrm{~cm}$ identificou a presença de 1807 indivíduos, equivalente a 221 indivíduos por hectare, com diâmetro médio de $15,5 \mathrm{~cm}$.

As estimativas de volume, biomassa e carbono alcançaram valores médios de $45,0 \mathrm{~m}^{3} / \mathrm{ha}, \quad 40,0$ t/ha e 20,0 t/ha, respectivamente. Embora a presença da espécie na área não ocorra em monodominância, verificou-se que a mesma apresenta produção em volume, biomassa e carbono superiores a outros fragmentos com a presença da espécie.

\section{REFERÊNCIAS}

BRASIL. Instituto Brasileiro de Geografia e Estatística. Manual técnico da vegetação brasileiras. Rio de Janeiro: IBGE, 2012. 275p.

BRASIL. Ministério do Meio Ambiente. INSTRUÇÃO NORMATIVA N ${ }^{\circ} 06$ de 23 de setembro de 2008. Traz a lista oficial de espécies da flora brasileira ameaçadas de extinção e com deficiência de dados. Diário Oficial da União,Poder Executivo, Brasília, DF, 24 set. 2008. Seção 1. p. 75.

BRUZINGA, J.S.; OLIVEIRA, M.L.R.; PEREIRA, I.M.; RABELO, L.D.O.; MURTA JÚNIOR, L.S.; DUARTE, A.L.A. Estoque de carbono em floresta estacional semidecidual em regeneração. Biomassa \& Energia, Viçosa, v. 5, n. 1, p. 77-83, 2012.

CARVALHO, P.E.R. Espécies arbóreas brasileiras. Brasília: Embrapa Florestas, 2003.1040p.

CARLINI, E.A.; DUARTE-ALMEIDA, J.M.; RODRIGUES, E.; PABACH, R. Antiulcer effect of the pepper trees Schinus terebinthifolius Raddi (aroeira-da-praia) and Myracrodruon urundeuva Allemão, Anacardiaceae (aroeira-dosertão). Revista Brasileira de Farmacognosia, Curitiba, v.20, n. 2, p. 140-146, 2010.

DRAPER, N.; SMITH, H. Applied regression analysis. New York: John Wiley \& Sons, 1998. 736p.

EMBRAPA - EMPRESA BRASILEIRA DE PESQUISA AGROPECUÁRIA. Sistema brasileiro de classificação de solos. Brasília: SPI/ CNPS, 1999. 412p.

FERNANDES, A. Biodiversidade da caatinga. In: ARAÚJO, E. L.; MOURA, A.N.; SAMPAIO, E.V.S.B.; GESTINARI, L.M.S.; CARNEIRO, J.M.T. (Eds.). Biodiversidade, conservação e uso sustentável da flora do Brasil. Recife: UFRPE/SBB, 2002. p. 42-43.

GOOGLE EARTH. Satellite's Image Software: versão 6.0.3.2197 2011. Disponível em <http://earth.google.com>. Acesso em: 14 out. 2013. 
GUARIM NETO, G. Plantas ornamentais de Mato Grosso. Boletin FBCN, Rio de Janeiro, v. 21, p. 105-115, 1986.

INMET - INSTITUTO NACIONAL DE METEOROLOGIA. BDMEP - Banco de Dados Meteorológicos para Ensino e Pesquisa: Série Histórica - Dados Diários de 01/01/1982 a 31/12/2012 Estação: 83464 JATAI - GO. Disponível em: <http://www.inmet.gov.br/portal/index.php?r=b dmep/bdmep>. Acesso em: 11 dez. 2013.

LORENZI, H. Árvores brasileiras - manual de identificação e cultivo de plantas arbóreas nativas do Brasil.Nova Odessa:Plantarum, 2002. $368 \mathrm{p}$.

MACHADO, S.A.; URBANO, E.; CONCEIÇÃO, M.B.; FIGUEIREDO FILHO, A.; FIGUEIREDO, D.J. Comparação de modelos de afilamento do tronco para diferentes idades e regimes de desbaste em plantações de Pinus oocarpa Schiede. Boletim de Pesquisa Florestal, Colombo, v. 48, p. 41-64, 2004.

MACHADO, S.A.; FIGUEIREDO FILHO, A. Dendrometria. 2ed. Guarapuava: UNICENTRO, 2009. 316p.

MACHADO JÚNIOR, J.A. Estudo de um fragmento com monodominância de aroeira (Myracroduon urundeuva Fr. All.) no Vale do Rio Doce-MG. 2007. 44f. Monografia (Graduação em Engenharia Florestal) Universidade Federal de Viçosa, Viçosa, MG.

OLIVEIRA, F.P. Monodominância de aroeira: fitossociologia, relações pedológicas e distribuição espacial em Tumiritinga - MG. 2011. 80 f. Dissertação (Mestrado em Solos e Nutrição de Plantas) - Universidade Federal de Viçosa, Viçosa, MG.

PAULA, J.E.; ALVES, J.L.H. Madeiras nativas: anatomia, dendrologia, dendrometria, produção e uso. Brasília: Fundação Mokiti Okada, 1997. 543p.

ROCHA, C.C.C. Estimativa de volume, biomassa e carbono da vegetação lenhosa de floresta estacional no estado de Goiás. 2011. 80f. Dissertação (Mestrado em Ciências
Florestais) - Universidade de Brasília, Brasília, DF.

SANTIN, D.A.; LEITAO FILHO, H.F.

Reestabelecimento e revisão taxonômica do gênero Myracrodruon Freire Allemão (Anacardeaceae). Revista Brasileira de Botânica, São Paulo,v. 14, n. 2, p. 133-145, 1991.

SEMA. Secretaria de Estado do Meio Ambiente. PORTARIA No. 99, de 20 de agosto de 2007.

Diário Oficial do Estado do Mato Grosso, Poder Executivo,Cuiabá, MT, 20 ago. 2007. p. 11.

SIQUEIRA, H.F.; CANGUSSU, J.T.; GUIMARÃES JÚNIOR, J.B.; ANDRADE, C.R. Determinação da densidade básica de Myracrodruon urundeuva. In: CONGRESSO BRASILEIRO DE CIÊNCIA E TECNOLOGIA DA MADEIRA, I.; SIMPÓSIO DE CIÊNCIA E TECNOLOGIA DO ESTADO DO RIO DE JANEIRO, III. Petrópolis. Anais... Seropédica: UFRRJ, 2013.

SCOLFORO, J.R.S. Manejo florestal. Lavras: UFLA/FAEPE, 1998. 443p.

SCOLFORO, J.R.S.; THIERSCH, C.R.

Biometria florestal: medição, volumetria e gravimetria. Lavras: UFLA/FAEPE, 2004. 285p.

SCOLFORO, J.R.S.; OLIVEIRA, A.D.; ACERBI JUNIOR, F.W. Inventário florestal de Minas Gerais: equações de volume, peso de matéria seca e carbono para diferentes fitofisionomias da flora nativa.Lavras: UFLA, 2008, 216p.

SOARES, C.P.B.; PAULA NETO, F.; SOUZA, A.L. Dendrometria e inventário florestal. Viçosa: UFV, 2006. 276p. 